\title{
Single-mode graded index fiber directional coupler : analysis by a simple and accurate method
}

\author{
S Sanyal, S Gangopadihyay ${ }^{+}$and S N Sarkar*

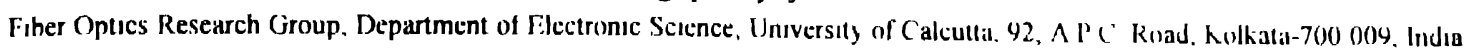 \\ 'Department of Physics. Surendranath ('ollege, '4 2 M (i Ruad, Kolkata-700) 00)9, India \\ E-maul sns(a)cucc ernet m
}

\begin{abstract}
Ibstract We present simple and accurate analysis of coupling characteristics of identical single-mude graded inder fiber directional couplers Hased on the recently developed simple series expression for the fundamental mode of such a filter involving. ('hebyshev lechnique

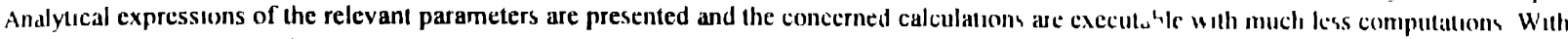
examples of step and parabolic index fiber directional couplers we show that our estımations match evecllently with the exact results
\end{abstract}

Keyuords Single-mode fibers, directional couplers.

P.ACS No. $4281 \mathrm{Qb}$

\section{Introduction}

Single-mode fiber directional couplers have emerged as the mosi prospective device in optical-fiber sensors [1-3], wavelength filters $[4]$ and nonlinear optical fiber based devices [5] ctc.. One should, therefore, know the coupling parameters accurately in order to prescribe its appropriate design in each field of use. Coupled-mode theory [6] has alteady been found to estimate accurately the coupling characteristics of step index fiber directional coupler over the pracucal range of parameters [7]. Further, during fabrication of single-mode step index fiber directional coupler, it becomes very difficult to maintain the step nature of the refractive Index profilc and therefore, the study of graded index fiber directional coupler is of tremendous importance.

The application of coupled-mode theory requires the knowledge of modal field distribution of each single-mode graded index fiber when they are non-interacting. In order to find the fundamental modal field for a single-mode graded index fiber one has to employ either numerical techniques or appropriatc methods excepting the case of step index fiber where analytical expressions are easily available. The Caussian approximation provides the simplest method in this context but as it fails to predict the cladding field accurately, it is not judicious to apply it for the analysis of fiber coupling

\footnotetext{
Corresponding Author
}

device. The variational technique involving two parameter Gaussian approximation $[8]$ has heen shown to estimate excellently the coupling features of step index fiber directional coupler. Again the variatıonal technique involving Gaussianexponential-H lankel function $[9,10]$ has been employed to predict accurately the coupling characteristics of singlemode parabolic core fiber directional coupler. These analyses, however, involve extensive computations. Therefore, prescription of a simple but accurate method in this connection is very necessary. Based on simple power series expression for the fundamental mode of graded index fiber [11] we quantify graded index fiber directional coupler over a wide and practical single-mode range in a simple but accurate manner.

\section{Theory}

For a directional coupler formed between a pair of identical graded index fibers, the refractive index corresponding to the coupled structure can be expressed as :

$$
\begin{aligned}
n^{2}(R) & =n_{1}^{2}\left[1-\delta R_{1}^{q}\right] & & 0<R_{1}<1, \\
& =n_{1}^{2}\left[1-\delta R_{2}^{4}\right] & & 0<R_{2}<1, \\
& =n_{1}^{2}[1-\delta]=n_{2}^{2}, & & \text { otherwise, }
\end{aligned}
$$


where $R_{1}-r_{1} / a$ and $R_{2}=r_{2} / a$ with $r_{1}$ and $r_{2}$ being the radial distance measured from the centres of the two fibers. $q$ is the profile exponent, which defines the shape of the profile. Under the condition of weak coupling, the coupled mode theory leads to the following expression for coupling coefficient $(C)[6,10 \mid$ given by :

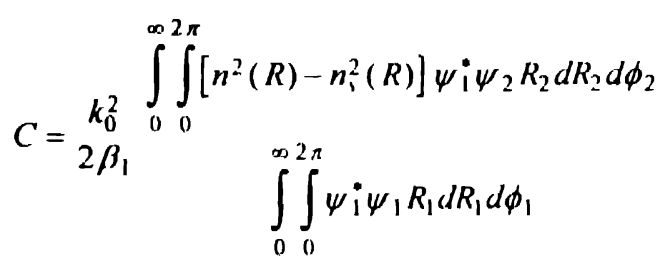

where $k_{0}$ is the free space wave number. $\psi_{1}$ and $\psi_{2}$ are the fundamental modes for respective fiber in isolation with $\beta_{\mathrm{i}}$ being the propagation constant in the each fiber.

Based on Chebyshev technique, it has been recently shown [11] that the transverse field of fundamental mode of graded index fiber can be accurately given :

$$
\begin{aligned}
a_{0}\left(1+A_{2} R^{2}+A_{4} R^{4}+A_{6} R^{6}\right), & R \leq 1 \\
(R)=a_{0}\left(1+A_{2}+A_{4}+A_{6}\right) \frac{K_{0}\left(W_{c}^{\prime} R\right)}{K_{0}\left(W_{c}\right)}, & R>1
\end{aligned}
$$

where $W_{c}$ is the value of $W$ found by the said Chebyshev formalism. If is relevant to mention that for a given value of $V$ one can easily find $W$ and the corresponding constant $A_{2 j}(\mathrm{~V}=1,2,3)$ with very little computations.

Using eq. (3) in eq. (2), we evaluate $C$ in case of step index fiber $(q=\infty)$ as follows :

$$
\begin{aligned}
C & =\frac{k_{0}^{2}\left(n_{1}^{2}-n_{2}^{2}\right)}{\beta_{1}} \\
& \times \frac{K_{0}\left(W_{c} d / a\right)}{K_{0}\left(W_{c}\right)} \frac{S_{2}\left[S_{9} I_{1}\left(W_{c}\right)-S_{10} I_{0}\left(W_{c}\right)\right]}{\left[S_{1}+S_{2}^{2}\left\{\frac{K_{1}^{2}\left(W_{c}\right)}{K_{0}^{2}\left(W_{c}\right)}-1\right.\right.},
\end{aligned}
$$

where $d$ represents the separation between the cores of the fibers and

$$
\begin{aligned}
\cdot S_{4} & =1+A_{2}^{2} / 3+A_{4}^{2} / 5+A_{6}^{2} / 7+A_{2}+2 A_{4} / 3+A_{6} / 2 \\
& +A_{2} A_{4} / 2+2 A_{2} A_{6} / 5+A_{4} A_{6} / 3, \\
S_{2} & =1+A_{2}+A_{4}+A_{6}, \\
S_{3} & =1 / W_{c}^{\prime}+4 / W_{c}^{2}, \\
S_{4} & =2 / W_{c}^{2}, \\
S_{5} & =1, W_{1}+16 / W_{c}^{3}+64 / W_{c}^{5}, \\
S_{6} & =4 / W_{c}^{2}+32 / W_{c}^{4}, \\
S_{7} & =1 / W_{\mathrm{c}}+36 / W_{c}^{1}+576 / W_{c}^{5}+2304 / W_{1}^{7}, \\
S_{8} & =6 / W_{c}^{2}+144 / W_{c}^{4}+1152 / W_{c}^{\prime}, \\
S_{9} & =1 / W_{c}+A_{2} S_{3}+A_{4} S_{9}+A_{6} S_{7}, \\
S_{10} & =A_{2} S_{4}+. A_{4} S_{6}+A_{6} S_{8} .
\end{aligned}
$$

Further, in case of parabolic index fiber $(q-2)$, the coupling coefficient is found by employing eq. (3) in eq. (2)

$$
\begin{aligned}
C= & \frac{k_{0}^{2}\left(n_{1}^{2}-n_{2}^{2}\right)}{\beta_{1}} \\
& \frac{K_{0}\left(W_{c} d / a\right)}{K_{0}\left(W_{c}\right)} \frac{S_{2}\left[S_{13} I_{1}\left(W_{c}\right)-S_{14} I_{0}\left(W_{c}\right)\right]}{\left.\left.S_{1}+S_{2}^{2}\left\{\begin{array}{l}
K_{1}^{2}\left(W_{c}\right) \\
K_{0}^{2}
\end{array} \frac{\left(W_{c}\right)}{(W)}\right\}\right]\right]},
\end{aligned}
$$

where $S_{11}=1 / W_{c}+64 / W_{c}^{3}+2304 / W_{c}^{5}$

$$
\begin{gathered}
\quad+36864 / W_{c}^{7}+147456 / W_{c}^{9}, \\
S_{12}=8 / W_{c}^{2}+384 / W_{c}^{4}+9216 / W_{c}^{6}+73728 / W_{c}^{\mathrm{g}}, \\
S_{13}=1 / W_{c}+A_{2} S_{3}+A_{4} S_{5}+A_{6} S_{7}-A_{2} S_{5}-A_{4} S_{7} \cdots A_{6}, S_{1 ;} \\
S_{14}=A_{2} S_{4}+A_{4} S_{6}+A_{6} S_{8}-S_{4}-A_{2} S_{6}-A_{4} S_{8}-A_{6} S_{1 ?}
\end{gathered}
$$

\section{Results and discussion}

In order to show the accuracy of our formulations for coupling coefficient we compare our results with the exact results in case of step as well as parabolic index fiber directional coupler. In Figure 1, we have plotted coupling coefficient $C$ (on a $\log _{10}$ scale) against the normarised centre.

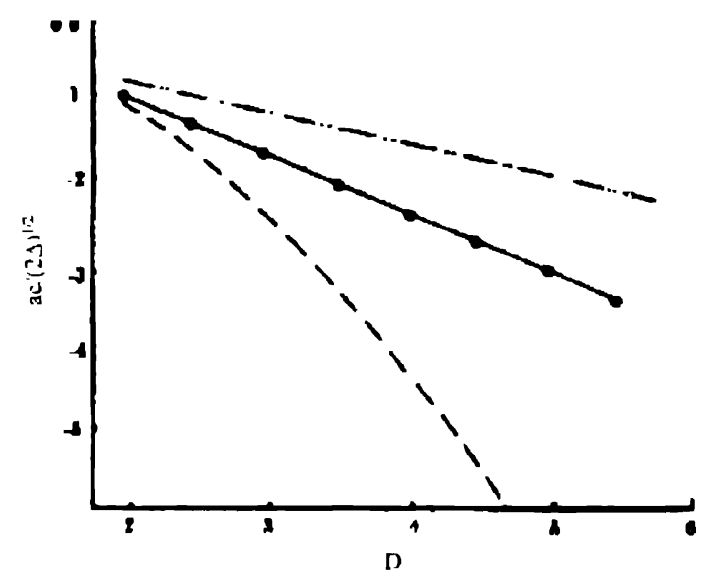

Figure 1. Variation of nonmalised scalar coupling coefficient \& with normalised separation $D$ for step core fiber Fiber directional couple" having $r=22$ (o 0 o our results, ---- Gaussian approxımatıons, .- exact results, - - modified Gaussıan approxımation)

to-centre core separation $D(=d / a)$ for a step index fiber directional coupler for $V=2.2$. It is seen that our predicuons virtually remain indistinguishable from the exact ones In this way, the superiority of our formalism over Gaussian and modified Gaussian methods [8] is established.

It is relevant to mention that though the modificd Gaussian method can quantify the step index fiber directional coupler quite accurately but it involved extensive computations while our formalism in this context require very little computations. Further, following Thyagarajan and Tewari [10] we also depict in Figure 2, the variation of normalised coupling length $\left(\tilde{L}_{c}=L_{c} \delta^{1 / 2} / a\right)$ with normalised separation $(D)$ for a parabolic index directional coupler for 
four different $V$ values namely $V=2.0, V=2.5, V=3.0$ and $f-3.5$. It is seen that our estimations agree excellently for dll said $V$ values in the entire range of $D$ values. Application

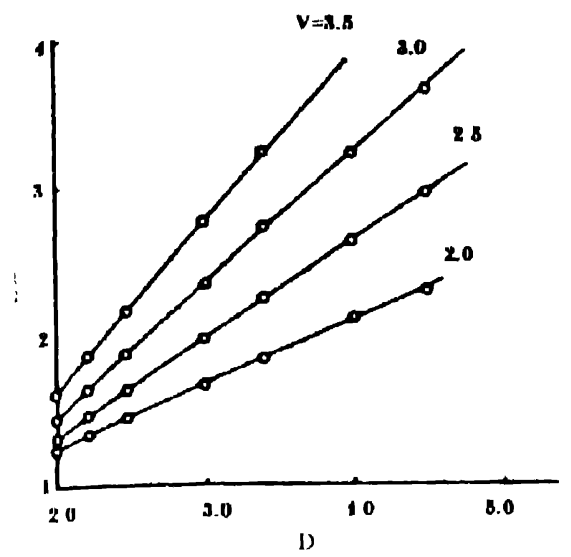

Loure 2. Vartation of normalised coupling length with nomalised (Epardien l) lor parabolic core liber directional coupler (o 0 o our results. eract results)

of Ciaussian-exponential-Hankal function also produces .tcurate results for the present problem but it requires quite Involved calculations of exponential integral functions. On we wher hand, our simple formalism quantifies the singlemode parabolic index fiber directional coupler excellently with very little computations. Accordingly, our formalism lunuld invite attention of system users because of its ilinplicity

\section{Conclüsions}

in present sumple analytical formulations for the analysis (1) coispling coeflicient of fiber directional coupler niade of two identical single-mode graded index fibers. With examples of step and parabolic index fiber directional couplers, we show that predictions by our simple formalism agrec excellently with the exact ones. The concerned calculations require very little computations.

\section{Acknowledgment}

The financial assistance of All India Council of Technical Education. India is gratefully acknowledged.

\section{Referencess}

[1] B Budauski, D C (Drucker, G S Kino and I R Rice Appl ()pt 184085 (1979)

[2] G B llocker Appl Opt 181445 (1979)

13] O Partiaus, G Chartice and I. Bernoux Kiber Optic Kotation Seit,ors and Relate'd 7echnologıes (Berlın, (jernanı Springer Verlag) (1982)

[4] M Digounet and II I Shaw Appl Ope 22484 (1983)

[5] (1 I Stcgeman and E. M Wright Opt Quantum Electron 2295 (1990)

16] A W Snyder and J D love Optical Wavegurde Theory (London (hapman and Hall) (1983)

171 I Eyges and P Wintersteiner $J$ Opt Soc Am $71 \quad 1351$ (1981)

[8] (j D) Peng and A Ankiewic/ J Modern Opt 382423 (1991)

[9] A Sharma, SI llossain and A K Ghatak Opt Quantum Electron 147 (1982)

[10] K Thyagarajan and R I cwan IEEE .J Light Tech LT-3 59 (1985)

[11] S (iangopadhyay and S N Sarhar Int ./ Optoelectron 11285 (1997) 